\title{
Effective polynomial upper bounds to perigees and numbers of $(3 x+d)$-cycles of a given oddlength
}

\author{
by
}

\author{
EDWARD G. Belaga (Strasbourg)
}

1. Introduction. Let $d$ be a positive odd integer not divisible by 3 , and let $T_{d}$ be the function defined on the set of positive integers, as follows: for all $m \in \mathbb{N}$,

$$
T_{d}(m)= \begin{cases}(3 m+d) / 2 & \text { if } m \text { is odd }, \\ m / 2, & \text { otherwise. }\end{cases}
$$

Repeated iterations of the function $T_{d}$ generate $(3 x+d)$ - (or $\left.T_{d^{-}}\right)$trajectories

$$
\tau_{d}(m)=\left\{m, T_{d}(m), T_{d}^{2}(m), \ldots\right\}
$$

for all $d \in \mathbf{D}=\{1,5,7,11,13, \ldots\}$ and $m \in \mathbb{N}$. By definition, a trajectory $\tau_{d}(m)$ is a cycle of length $l, \mathbf{C}=\mathbf{C}(m, d)=\tau_{d}(m)$, length $(\mathbf{C})=l$, if $T_{d}^{l}(m)=$ $m$ and, for any $j \in[1, l-1], T_{d}^{j}(m) \neq m$ (note that $l>1$, since the mapping $T_{d}$ has no fixed points). The minimal member of a $T_{d}$-cycle $\mathbf{C}$ is odd, and is called its perigee, $n_{0}=\operatorname{prg}(\mathbf{C})$. Thus, the number $k$ of odd members of a $T_{d}$-cycle, called here its oddlength, is a positive integer, $k \geq 1$. The length and oddlength of a cycle are related by the inequality $l \geq\left\lceil k \log _{2} 3\right\rceil$ [Belaga, Mignotte 1998] (see Theorem 3.2(1) below). Note also that no member of a $T_{d}$-trajectory (1.2), excluding possibly the first one, is divisible by 3 , and thus, all odd members of a $T_{d}$-cycle belong to $\mathbf{D}$.

It has been conjectured that the dynamical system $\mathcal{D}_{d}=\left\{\mathbb{N}, T_{d}\right\}$ has

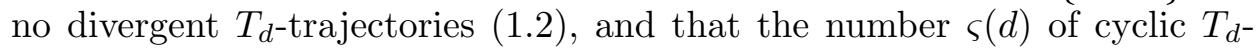
trajectories is finite [Lagarias 1990], [Belaga, Mignotte 1998]. In the particular case $d=1$, the well-known $3 x+1$ conjecture [Lagarias 1985], [Wirsching $1998]$ is even more specific: any trajectory $\tau_{1}(m)$ enters ultimately the (only) $3 x+1$ cycle $\{1 \rightarrow 2 \rightarrow 1\}$. $11 \mathrm{~B} 85$.

2000 Mathematics Subject Classification: Primary 11K31, 11K38, 11K55; Secondary

Key words and phrases: $3 x+1$ and $3 x+d$ functions, $3 x+1$ and $3 x+d$ conjectures, divergent trajectory, cycle, perigee, length, oddlength, odd frame, Collatz signature of a cycle. 
The present paper is concerned with the cyclic part of the above $3 x+d$ conjecture, and more generally, with quantitative (and when available, numerical) characteristics of the cyclic structure of systems $\mathcal{D}_{d}$. Let $\mathcal{C}(d)$ and $\mathcal{C}_{k}(d)$ be the sets of all $T_{d}$-cycles and, respectively, of all such cycles with $k$ odd members, or, in our terminology, of oddlength $k \geq 1$.

Technically, our main result is the following general upper bound on the perigee of a $T_{d}$-cycle of length $l$ and oddlength $k$ : for all $d \in \mathbf{D}$ and $\mathbf{C} \in \mathcal{C}(d)$,

$$
\left\{\begin{array}{l}
\operatorname{length}(\mathbf{C})=l \\
\text { oddlength }(\mathbf{C})=k
\end{array}\right\} \Rightarrow n_{0}=\operatorname{prg}(\mathbf{C}) \leq \frac{d}{2^{l / k}-3} .
$$

The inequality (1.3) has four important implications.

The first one is an upper bound on the ratio of the length of a $T_{d^{-} \text {-cycle }}$ to its oddlength, which, together with the well-known lower bound (2.5), Theorem 2.1(1), confines this ratio to the interval:

$$
\log _{2} 3<\varrho_{d}(\mathbf{C})=\frac{\text { length }(\mathbf{C})}{\text { oddlength }(\mathbf{C})} \leq \log _{2}(d+3) .
$$

The upper bound is sharp, and so is, in all probability, the lower bound; but the considerations leading to the corresponding conclusions are quite different in nature.

Consider first the case of the upper bound. For any $r \geq 2$, the $T_{2^{r}-3^{-c y c l e}}$ $\mathbf{C}_{2^{r}-3}^{0}$ of length $r+1$, starting at (the odd number) 1 , has no other odd members:

$$
\begin{gathered}
\mathbf{C}_{2^{r}-3}^{0}=\left\{1,2^{r-1}, 2^{r-2}, \ldots, 2\right\} ; \\
\text { oddlength }\left(\mathbf{C}_{2^{r}-3}^{0}\right)=1 ; \quad \text { length }\left(\mathbf{C}_{2^{r}-3}^{0}\right)=r=\log _{2}\left(\left(2^{r}-3\right)+3\right) .
\end{gathered}
$$

As to the lower bound, the calculations carried out in [Belaga, Mignotte 2000] (e.g., there exists a $T_{233}$-cycle starting at 919 , of length 13 and oddlength $\left.8,1.584<\log _{2} 3<1.585<1.625=13 / 8\right)$ show the high plausibility of the following conjecture:

Conjecture 1.1. For any $\varepsilon>0$, there exist a triplet of positive integers, $d \in \mathbf{D},(k, l) \in \mathbb{N}^{2}, \log _{2} 3<l / k<\log _{2} 3+\varepsilon$, and a $T_{d^{-}}$cycle of length $l$ and oddlength $k$.

Cf. also the inequalities (1.12) below.

Second, the inequality (1.3) implies the following general and uniform upper bound on the perigees of $T_{d}$-cycles of oddlength $k \geq 1$ :

$$
n_{0}=\operatorname{prg}(\mathbf{C}) \leq \mathbf{U}_{d, k}=\frac{d}{2^{\left\lceil k \log _{2} 3\right\rceil / k}-3} .
$$

The bound (1.5) has an effective polynomial numerical equivalent (see the estimate (1.9) below). It is also sharp in the following natural sense (Theorem $3.2,(3.11)(1))$ : the average value of an odd member of a $T_{d}$-cycle of 
the oddlength $k \geq 1$ is bigger than $\mathbf{U}_{d, k}$. Thus, for example, the $T_{5}$-cycle $\mathbf{C}=\{23 \rightarrow 37 \rightarrow 58 \rightarrow 29 \rightarrow 46\}$ has 3 odd members, $n_{0}=\operatorname{prg}(\mathbf{C})=23<$ $\mathbf{U}_{5,3} \approx 28.6038<29<37$.

Third, since no two $T_{d}$-cycles have a common member, any such cycle is fully determined by its perigee. Thus, the upper bound (1.5) not only implies that the set $\mathcal{C}_{k}(d)$ of $T_{d}$-cycles of oddlength $k \geq 1$ is finite, but supplies us with an effective general upper bound on the number $\varsigma_{k}(d)=\# \mathcal{C}_{k}(d)$ of $T_{d}$-cycles of oddlength $k$ :

$$
\varsigma_{k}(d) \leq \frac{1}{3} \mathbf{U}_{d, k}=\frac{1}{3} \cdot \frac{d}{2^{\left\lceil k \log _{2} 3\right\rceil / k}-3}=\frac{1}{9} \cdot \frac{d}{2^{\left(\left\lceil k \log _{2} 3\right\rceil-k \log _{2} 3\right) / k}-1}
$$

(the factor $1 / 3$ is due to the aforementioned inclusion $n_{0} \in \mathbf{D}$ ).

Any numerical evaluation of the expression $\mathbf{U}_{d, k}$ depends on our knowledge of effective lower bounds for diophantine approximations of linear combinations of $\log a r i t h m s \log 2$ and $\log 3$ (cf. the left inequality in (1.4)),

$$
\varepsilon_{k}=\left\lceil k \log _{2} 3\right\rceil-k \log _{2} 3=\frac{1}{\log 2} \min _{l>k \log _{2} 3}(l \log 2-k \log 3) .
$$

According to [Baker, Wüstholz 1993], for some effectively calculable constant $C_{1}>0$, we have:

$$
\forall k, l \in \mathbb{Z}, k<l, \quad|l \log 2-k \log 3|>k^{-C_{1}} .
$$

One easily deduces from (1.8) the existence of an effectively calculable constant $C_{2}>0$ such that for all $d \in \mathbf{D}$ and $k>2$,

$$
\mathbf{U}_{d, k} \leq d k^{C_{2}} \text {. }
$$

The original bound [Baker, Wüstholz 1993] on the constant $C_{1}$ (and thus, of the closely related $C_{2}$ ) has been enormous. Using less general but more appropriate techniques (linear combination of only two logarithms) of [Laurent et al. 1995, Corollary 2], one can easily reduce the value $C_{2}$ to a two-digit number, $C_{2}<32$.

Fourth, as is clear from the right side expressions of the upper bounds (1.3), (1.5), (1.6), the values of pairs $(k, l)$ corresponding to potentially "rich" or "numerous" families of $d$-cycles do not actually depend on $d$ (which enters all three expressions as a linear factor) but only on how close to zero the value $|l \log 2-k \log 3|$ is.

Thus, any result concerning (non-)existence of $d$-cycles, for a specific value of $d$, of oddlength $k$ and length $l$ would probably imply, or at least strongly hint at, the (non-)existence of $d^{\prime}$-cycles, for all $d^{\prime} \in \mathbf{D}$, as well.

Historical remarks. The present author is not aware of any previous effective (and in any sense sharp) upper bound on the minimal odd member of a $T_{d}$-cycle. The following general exponential upper bound on the number $\varsigma_{k}(d)$ of $T_{d}$-cycles of oddlength $k \geq 1$ was actually (implicitly) proved in 
[Belaga, Mignotte 1998] and refined in [Belaga, Mignotte 2000]: for all $d \in \mathbf{D}$ and $k \in \mathbb{N}$,

$$
\varsigma_{k}(d)<d\left(\frac{3}{2}\right)^{k} \frac{2^{\varepsilon_{k}}}{2^{\varepsilon_{k}}-1}, \quad \varepsilon_{k}=\left\lceil k \log _{2} 3\right\rceil-k \log _{2} 3 .
$$

The bound (1.10) was derived from an identical upper bound on the maximal odd member of a cycle, the corresponding numerical upper bound being based on the aforementioned estimate of [Baker, Wüstholz 1993]: for all $d \in \mathbf{D}$ and $k \in \mathbb{N}$,

$$
\varsigma_{k}(d)<d k^{C}\left(\frac{3}{2}\right)^{k}
$$

Comments and future prospects. (1) The upper bound (1.4) on the ratio $\varrho_{d}(\mathbf{C})$ implies in the $3 x+1$ case that the length of a cycle with $k$ odd members does not exceed $2 k$. Note that the only $3 x+1$ cycle known at present, $\{1 \rightarrow 2 \rightarrow 1\}$, has one odd member, is of length two, and has ratio two. A slightly more elaborate argument (to be published elsewhere) shows that the length and oddlength of any other $3 x+1$ cycle (in case it exists) should satisfy the inequalities:

$$
1.584<\log _{2} 3 \leq \varrho_{1}(\mathbf{C}) \leq 4-\log _{2} 5<1.679 .
$$

(2) The bounds (1.5) and, especially, (1.6) can be apparently improved. In fact, the experimental discovery of $843 T_{14303}$-cycles of oddlength 17 , with perigees varying from 385057 to $1391321<\mathbf{U}_{14303,17}=2099280$, suggests that the bound (1.5) is apparently sharp up to a one-digit constant.

As to the bound (1.6), our calculations have unearthed 944 different $T_{14303}$-cycles of oddlengths, respectively, $k=17$ (843 cycles), 34 (76), 51 (20), 68 (3), 85 (1), 1092 (1), implying the inequality

$$
843 \leq \varsigma_{17}(14303)<\frac{1}{3} \mathbf{U}_{14303,17}=699760 .
$$

This estimate, far from being sharp, is at least realistic: for some $d, k$, the dynamical system $\mathcal{D}_{d}$ has "many" cycles of oddlength $k$.

(3) At present, the bounds (1.3), (1.5), (1.6) look useless, or at least insufficient, for a possible proof of the cyclic part of the $3 x+d$ conjecture, i. e., of the finiteness of the number $\varsigma(d)$ of $T_{d}$-cycles.

However, this obstacle could possibly be circumvented by a refinement of the above scheme, to fit the purpose of yielding directly an absolute (i.e., not depending on $k$ ) upper bound on the number $\varsigma(d)$.

Acknowledgements. The anonymous referee expressed his reservations about the sufficiency of the argument leading to the above upper bound (1.9) (in the first version of the present paper, with a different effective con- 
stant $C_{2}$ ). His insight was completely justified, and the above derivation of (1.9) with $C_{2}<32$ from Corollary 2 of the paper [Laurent et al. 1995] is due to the author's discussions with Maurice Mignotte, one of the co-authors of the above paper.

2. Exponential diophantine formulae for $3 x+d$ cycles. Let, as above, $\mathbf{C}=\mathbf{C}(m, d)=\tau_{d}(m)$ be a $T_{d}$-cycle of length $l$, length $(\mathbf{C})=l$. We remind the reader that, according to (1.1), the minimal member, or perigee of a $T_{d^{-} \text {-cycle, }} n_{0}=\operatorname{prg}(\mathbf{C})$, is odd, and that the total number $k \geq 1$ of odd members of a cycle is called its oddlength. Moreover, if $n$ is an odd member of a cycle, then $n \in \mathbf{D}$ (see (1.2)), since no number divisible by 3 can belong to a cycle.

Note that if $m^{\prime} \neq m$ is a member of a $T_{d^{-}}$cycle $\mathbf{C}=\tau_{d}(m)$, or in other words, if $\mathbf{C}$ meets $m^{\prime}$, one should view $\mathbf{C}^{\prime}=\mathbf{C}\left(m^{\prime}, d\right)$ as just another name for the same cycle $\mathbf{C}=\mathbf{C}(m, d)$. Since a $T_{d^{-}}$cycle is fully characterized by its minimal member, the following notation can be adopted as the canonical one:

$$
\mathbf{C}=\mathbf{C}\left(n_{0}, d\right)=\mathbf{C}\left[n_{0}, d\right]=\tau_{d}\left(n_{0}\right), \quad n_{0}=\operatorname{prg}(\mathbf{C}) .
$$

In this case, we also say that $\mathbf{C}$ starts at $n_{0}$.

For any positive integer $m \in \mathbb{N}$, let odd $(m)$ be the number obtained by factoring out $m$ by the highest possible power of 2 , say $2^{j}$, and let $\nu_{2}(m)=j$. Thus odd $(m)$ is odd and $m=\operatorname{odd}(m) \cdot 2^{\nu_{2}(m)}$. Define

$$
S: \mathbf{D} \times \mathbf{D} \rightarrow \mathbf{D}, \quad(n, d) \mapsto S_{d}(n)=\operatorname{odd}(3 n+d) .
$$

The function $S_{d}$ speeds up the action of $T_{d}$, skipping even members of $T_{d^{-}}$ trajectories. In particular, $m=1$ becomes the fixed point of the function $S_{1}=\operatorname{odd}(3 n+1), S_{1}(1)=1$, corresponding to the (according to the $3 x+1$ conjecture, only) $T$-cycle $\mathbf{C}(1,1)=\{1 \rightarrow 2 \rightarrow 1\}$.

We associate with any $T_{d}$-cycle $\mathbf{C}=\mathbf{C}\left[n_{0}, d\right]$ its odd frame, $\mathbf{F}=\operatorname{Odd}(\mathbf{C})$, the list of odd members of the cycle, in the order of their appearance in $\tau_{d}\left(n_{0}\right)$, as the $T_{d}$-iterations of $n_{0}$ proceed. By definition, the frame is an

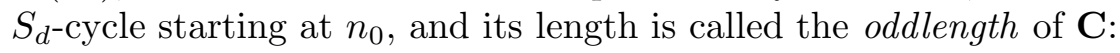

$$
\begin{aligned}
l & =\operatorname{length}(\mathbf{C})=\min \left\{i \in \mathbb{N} \mid m_{i}=T_{d}^{i}\left(n_{0}\right)=n_{0}\right\} ; \\
k & =\operatorname{oddlength}(\mathbf{C})=\min \left\{j \in \mathbb{N} \mid n_{j}=S_{d}^{j}\left(n_{0}\right)=n_{0}\right\} ; \\
\mathbf{F} & =\operatorname{Odd}(\mathbf{C})=\left\langle n_{0}, n_{1}, \ldots, n_{k-1}\right\rangle \in \mathbf{D}^{k} .
\end{aligned}
$$

The even members of the $T_{d}$-cycle $\mathbf{C}=\mathbf{C}\left[n_{0}, d\right]$ can be recovered from its frame with the help of the cycle Collatz signature $\mathbf{P}=\theta(\mathbf{C})$, the vector of exponents of 2 factoring out from the values of the function $T_{d}$ at odd members of $\mathbf{C}$, as follows: 


$$
\begin{aligned}
& \mathbf{F}=\operatorname{Odd}(\mathbf{C})=\left\langle n_{0}, n_{1}, \ldots, n_{k-1}\right\rangle ; \\
& \forall j \in[0, k-1], \quad p_{j+1}=\nu_{2}\left(T_{d}\left(n_{j}\right)\right)+1=\nu_{2}\left(3 n_{j}+d\right) ; \\
& \mathbf{P}=\theta(\mathbf{C})=\left\langle p_{1}, \ldots, p_{k}\right\rangle \in \mathbb{N}^{k} ; \\
& l=\operatorname{length}(\mathbf{C})=|\mathbf{P}|=p_{1}+\ldots+p_{k} ; \\
& \forall j \in[1, k-1],\left\{\begin{array}{l}
m_{p_{1}+\ldots+p_{j}}=n_{j} ; \\
p_{j}>1 \Rightarrow \forall i \in\left[1, p_{j}-1\right], m_{p_{1}+\ldots+p_{j}-i}=2^{i} n_{j} .
\end{array}\right.
\end{aligned}
$$

Moreover, the Collatz signature $\mathbf{P}=\theta(\mathbf{C})$ of a cycle $\mathbf{C}=\mathbf{C}\left[n_{0}, d\right]$, where $n_{0}, d \in \mathbf{D}$, completely characterizes it:

Theorem 2.1 [Belaga, Mignotte 1998]. (1) The Collatz signature $\mathbf{P}=$ $\theta(\mathbf{C})$ satisfies the inequality:

$$
l=|\mathbf{P}|=p_{1}+\ldots+p_{k} \geq\left\lceil k \log _{2} 3\right\rceil .
$$

(2) Define the exponential diophantine function $A=a_{k}: \mathbb{N}^{k} \rightarrow \mathbb{N}$, as follows: for $\mathbf{P}=\left\langle p_{1}, \ldots, p_{k}\right\rangle \in \mathbb{N}^{k}$,

$$
\begin{aligned}
& A=a_{k}(\mathbf{P}) \\
= & \begin{cases}1 & \text { if } k=1 ; \\
3^{k-1}+2^{p_{1}} \cdot 3^{k-2}+\ldots+2^{p_{1}+\ldots+p_{k-2}} \cdot 3+2^{p_{1}+\ldots+p_{k-1}} & \text { otherwise. }\end{cases}
\end{aligned}
$$

Let $\sigma=\sigma_{k}$ be the circular (counterclockwise) permutation on $k$-tuples: for $\mathbf{P}=\left\langle p_{1}, \ldots, p_{k}\right\rangle \in \mathbb{N}^{k}$

$$
\sigma(\mathbf{P})=\sigma_{k}(\mathbf{P})=\left\langle p_{2}, \ldots, p_{k}, p_{1}\right\rangle
$$

If now $\mathbf{P}=\theta(\mathbf{C})$ is the Collatz signature of a cycle $\mathbf{C}=\mathbf{C}\left[n_{0}, d\right], n_{0}, d \in \mathbf{D}$, of length $l$, oddlength $k \geq 1$, and with the frame $\mathbf{F}=\left\langle n_{0}, n_{1}, \ldots, n_{k-1}\right\rangle$, then

$$
\left\{\begin{array}{lll}
(1) & B=b_{k}(\mathbf{P})=B_{k, l}=2^{l}-3^{k}>0 & \text { (cf. (2.5)); } \\
(2) & n_{0}=d \frac{A}{B}, \quad A=a_{k}(\mathbf{P}) & \text { (cf. (2.6)); } \\
(3) \quad \forall j \in[1, k-1], \quad n_{j}=d \frac{a_{k}\left(\sigma^{j}(\mathbf{P})\right)}{B} & \text { (cf. (2.7)). }
\end{array}\right.
$$

3. Upper bound on the number of $3 x+d$ cycles of a given oddlength. According to the formulae $(2.8)(2),(3)$, the odd members of a $T_{d}$-cycle of oddlength $k$ satisfy the inequality

$$
n_{j} \leq \mathbf{W}_{d, k}=d \sup _{\substack{\mathbf{P} \in \mathbb{N}^{k} \\|\mathbf{P}| \geq k \log _{2} 3}} \frac{a_{k}(\mathbf{P})}{2^{|\mathbf{P}|}-3^{k}}=d \sup _{l \geq k \log _{2} 3} \frac{\max _{\mathbf{P} \in \mathbb{N}^{k},|\mathbf{P}|=l} a_{k}(\mathbf{P})}{2^{l}-3^{k}}
$$

for all $j \in[1, k-1]$. Simple calculations show that (cf. (1.10) above)

$$
\mathbf{W}_{d, k} \leq d\left(\frac{3}{2}\right)^{k} \frac{2^{\varepsilon_{k}}}{2^{\varepsilon_{k}}-1}, \quad \varepsilon_{k}=\left\lceil k \log _{2} 3\right\rceil-k \log _{2} 3
$$


We will be able to improve these bounds thanks, first, to a more careful analysis of the formulae (2.8), and then, to a remarkable inequality (3.5) proved below (Theorem 3.1). Namely, instead of evaluating from above all members of a $T_{d}$-cycle of oddlength $k$, we evaluate here its minimal member

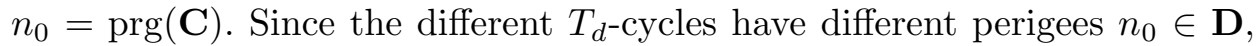
an upper bound $n_{0} \leq \mathbf{V}_{d, k}^{\prime}$ would imply the bound $\varsigma_{k}(d) \leq \frac{1}{3} \cdot \mathbf{V}_{d, k}^{\prime}$ to the number of $T_{d}$-cycles of the oddlength $k$.

More formally, if $\mathbf{P}=\theta(\mathbf{C})$ (2.4) is the Collatz signature of the cycle $\mathbf{C}=\mathbf{C}\left[n_{0}, d\right], n_{0}, d \in \mathbf{D}$, of length $l=|\mathbf{P}|$ and oddlength $k \geq 1$, then, according to $(2.8)$,

$$
n_{0} \leq \min \left\{n_{0}, n_{1}, \ldots, n_{k-1}\right\}=d \frac{\min _{j \in[0, k-1]}\left\{a_{k}\left(\sigma^{j}(\mathbf{P})\right)\right\}}{2^{l}-3^{k}} .
$$

For any $k$-tuple $\mathbf{P}$ of positive integers define its average $\overline{\mathbf{P}}$ to be the arithmetical mean of all its counterclockwise permutations. This $k$-tuple of positive (generally speaking, rational) numbers depends only on the dimension $k$ and length $l=|\mathbf{P}|$ of $\mathbf{P}$ :

$$
\overline{\mathbf{P}}=\frac{1}{k} \sum_{j \in[0, k-1]} \sigma^{j}(\mathbf{P})=\left\{\frac{l}{k}, \ldots, \frac{l}{k}\right\} .
$$

Extending the definition of the function $a_{k}$ (see (2.6)) to $k$-tuples of positive reals, we will prove below (Theorem 3.2) the inequality

$$
\widetilde{a}_{k}(\mathbf{P})=\min _{j \in[0, k-1]}\left\{a_{k}\left(\sigma^{j}(\mathbf{P})\right)\right\} \leq a_{k}(\overline{\mathbf{P}})=\frac{2^{l}-3^{k}}{2^{l / k}-3}
$$

for $\mathbf{P} \in \mathbb{N}^{k}$. The inequalities (3.3) and (3.5) imply the general upper bound (1.5), depending only on $d$ and $k$, for the minimal member $n_{0}=\operatorname{prg}(\mathbf{C})$ of any $T_{d}$-cycle of oddlength $k$ :

$$
\forall n, d \in \mathbf{D}, \quad \mathbf{C}=\mathbf{C}[n, d] \Rightarrow n \leq \mathbf{U}_{d, k}=\frac{d}{2^{l / k}-3},
$$

and, finally, the upper bound (1.6).

Definition 3.1. (1) Let $\Lambda$ be the set of pairs of positive integers $(k, l)$ satisfying the inequality implied by (2.5),

$$
\Lambda=\left\{(k, l) \in \mathbb{N}^{2} \mid \lambda(k, l)=l-\left\lceil k \log _{2} 3\right\rceil \geq 0\right\} .
$$

Extend the definition of the function $A=a_{k}$ (see (2.6)) to $k$-tuples of positive reals from the $(k-1)$-dimensional tetrahedron $\mathbf{T}_{k, l},(k, l) \in \Lambda$,

$$
\mathbf{T}_{k, l}=\left\{\mathbf{X} \in \mathbb{R}^{k}|| \mathbf{X} \mid=x_{1}+\ldots+x_{k}=l \wedge \forall j \in[1, k], x_{j} \geq 1\right\},
$$


with $k$ vertices $\mathbf{V}_{1}, \ldots, \mathbf{V}_{k}$,

$$
\begin{aligned}
\mathbf{V}_{1} & =\{l-k+1,1, \ldots, 1\}, \\
\mathbf{V}_{2} & =\{1, l-k+1, \ldots, 1\}, \ldots, \\
\mathbf{V}_{k} & =\{1,1, \ldots, l-k+1\} .
\end{aligned}
$$

(2) The permutation $\sigma$ (see (2.7)) induces on $\mathbf{T}_{k, l}$ the rotation $\sigma$, with the center $\mathbf{O}$ of the tetrahedron being the only fixed point: for $\mathbf{X}=\left\langle x_{1}, x_{2}, \ldots\right.$, $\left.x_{k-1}, x_{k}\right\rangle \in \mathbf{T}_{k, l}$,

$$
\begin{aligned}
& \sigma(\mathbf{X})=\left\langle x_{2}, x_{3}, \ldots, x_{k}, x_{1}\right\rangle ; \\
& \overline{\mathbf{X}}=\frac{1}{k} \sum_{j \in[1, k]} \sigma^{j}(\mathbf{X})=\left\langle\frac{l}{k}, \ldots, \frac{l}{k}\right\rangle=\mathbf{O} ; \\
& \sigma(\mathbf{O})=\mathbf{O} ; \\
& a_{k}(\mathbf{O})=\sum_{j=1}^{k} 3^{k-j} 2^{l / k}=\frac{2^{l}-3^{k}}{2^{l / k}-3} .
\end{aligned}
$$

Theorem 3.2. For any $k$-tuple $\mathbf{X}$ from $\mathbf{T}_{k, l}$, we have

$$
\left\{\begin{array}{l}
(1) \quad \bar{a}_{k}(\mathbf{X})=\frac{1}{k} \sum_{j=0}^{k-1} a_{k}\left(\sigma^{j}(\mathbf{X})\right) \geq a_{k}(\mathbf{O})=\frac{2^{l}-3^{k}}{2^{l / k}-3}, \\
(2) \quad \tilde{a}_{k}(\mathbf{X})=\min _{j \in[0, k-1]}\left\{a_{k}\left(\sigma^{j}(\mathbf{X})\right)\right\} \leq a_{k}(\mathbf{O})=\frac{2^{l}-3^{k}}{2^{l / k}-3},
\end{array}\right.
$$

with equalities holding only in the case $\mathbf{X}=\mathbf{O}$.

4. Proof of Theorem 3.2. Note that, according to (2.6), if $k=1$, then $l \geq 2$ and for $\mathbf{X} \in \mathbf{T}_{1, l}$,

$$
\overline{\mathbf{X}}=\mathbf{X}=\mathbf{O}, \quad \bar{a}_{k}(\mathbf{X})=\widetilde{a}_{k}(\mathbf{X})=\frac{2^{l}-3}{2^{l}-3}=1
$$

Thus, it can be henceforth assumed that $k \geq 2$.

(1) The inequality $(3.11)(1)$ is implied by the standard inequality $\frac{1}{k}(a+$ $b+\ldots) \geq \sqrt[k]{a \cdot b \cdot \ldots}$, as follows: for all $k \geq 2,(k, l) \in \Lambda$, and $\mathbf{X} \in \mathbf{T}_{k, l}$,

$$
\begin{aligned}
\frac{1}{k} \sum_{0 \leq j \leq k-1} a_{k}\left(\sigma^{j}(\mathbf{X})\right) & =3^{k-1}+\sum_{1 \leq j \leq k-1} \frac{3^{k-j-1}}{k} \sum_{0 \leq r \leq k-1} 2^{\sigma^{r}\left(x_{1}+\ldots+x_{j}\right)} \\
& \geq 3^{k-1}+\sum_{1 \leq j \leq k-1} 3^{k-j-1} \cdot 2^{\frac{1}{k} \sum_{0 \leq r \leq k-1} \sigma^{r}\left(x_{1}+\ldots+x_{j}\right)} \\
& =a_{k}(\mathbf{O})=\frac{2^{l}-3^{k}}{2^{l / k}-3} \quad(\mathrm{cf} .(3.10)) .
\end{aligned}
$$


(2) If $\mathbf{X}=\mathbf{O} \in \mathbf{T}_{k, l}$, then (3.11)(2) becomes a trivial identity. Otherwise, $\mathbf{O} \neq \mathbf{X} \in \mathbf{T}_{k, l}(k \geq 2,(k, l) \in \Lambda)$, and among the $k k$-tuples $\sigma^{j}(\mathbf{X}), 0 \leq$ $j \leq k-1$, there exist at least two different ones:

$$
\begin{array}{ll}
\forall j \in[0, k-1], & \sigma^{j}(\mathbf{X}) \neq \overline{\mathbf{X}}=\mathbf{O} ; \\
\exists j \in[1, k-1], & \mathbf{X} \neq \sigma^{j}(\mathbf{X}) .
\end{array}
$$

Now the proof proceeds ad absurdum: the assumption $a_{k}\left(\sigma^{j}(\mathbf{X})\right)>a_{k}(\mathbf{O})$ for all $j \in[0, k-1]$ would imply that $a_{k}(\overline{\mathbf{X}})>a_{k}(\mathbf{O})$ as well-a contradiction, since $\overline{\mathbf{X}}=\mathbf{O}$ (see (3.10), (4.2)).

The equation $a_{k}(\mathbf{X})=a_{k}(\mathbf{O})$ induces a break up of the $(k-1)$-dimensional tetrahedron $\mathbf{T}_{k, l}$ (see (3.8)) into three disjoint subsets: the closed $(k-2)$ dimensional submanifold $\mathbf{T}^{0}=\mathbf{T}_{k, l}^{0}$ defined by this equation, and two $(k-1)$ dimensional submanifolds $\mathbf{T}^{+}=\mathbf{T}_{k, l}^{+}$and $\mathbf{T}^{-}=\mathbf{T}_{k, l}^{-}$, open in $\mathbf{T}_{k, l}$, defined by the inequalities $a_{k}(\mathbf{X})>a_{k}(\mathbf{O})$ and $a_{k}(\mathbf{X})<a_{k}(\mathbf{O})$, respectively:

$$
\begin{aligned}
\mathbf{T}^{0} & =\mathbf{T}_{k, l}^{0}=\left\{\mathbf{X} \in \mathbf{T}_{k, l} \mid a_{k}(\mathbf{X})=a_{k}(\mathbf{O})=\left(2^{l}-3\right) /\left(2^{l / k}-3\right)\right\} \\
\mathbf{T}^{+} & =\mathbf{T}_{k, l}^{+}=\left\{\mathbf{X} \in \mathbf{T}_{k, l} \mid a_{k}(\mathbf{X})>a_{k}(\mathbf{O})\right\} \\
\mathbf{T}^{-} & =\mathbf{T}_{k, l}^{-}=\left\{\mathbf{X} \in \mathbf{T}_{k, l} \mid a_{k}(\mathbf{X})<a_{k}(\mathbf{O})\right\}
\end{aligned}
$$

Below we prove the following properties of these three submanifolds:

(A) $\mathbf{T}^{0}$ is a smooth (in fact, analytical) submanifold.

(B) The submanifolds $\mathbf{T}^{0}, \mathbf{T}^{+}, \mathbf{T}^{-}$are connected and simply connected.

(C) The closed set $\mathbf{T}^{0+}=\mathbf{T}^{0} \cup \mathbf{T}^{+}$is strictly convex: the convex closure $\mathcal{P}(S)$ of a finite set $S$ of $k$-tuples from $\mathbf{T}^{0+}$ is contained in $\mathbf{T}^{+}$, excluding, if necessary, the tuples from $S$ belonging to $\mathbf{T}^{0}$.

The last property immediately implies the validity of the above argument ad absurdum.

To prove $(\mathrm{A})-(\mathrm{C})$, one needs to look at the first and second partial derivatives of the function $a_{k}(\mathbf{X})$ : for all $k \geq 2,(k, l) \in \Lambda$ and $\mathbf{X}=\left\langle x_{1}, \ldots, x_{k}\right\rangle$ $\in \mathbf{T}_{k, l}$,

$$
\begin{aligned}
& a_{k}(\mathbf{X})=\left\{\frac{\partial a_{k}}{\partial x_{1}}, \ldots, \frac{\partial a_{k}}{\partial x_{k-1}}, \frac{\partial a_{k}}{\partial x_{k}}\right\}(\mathbf{X}) \\
& =\ln 2 \cdot\left\{2^{x_{1}} a_{k-1}\left(x_{2}, \ldots, x_{k}\right), \ldots, 2^{x_{1}+\ldots+x_{k-1}} a_{1}\left(x_{k}\right), 0\right\} ; \\
& \frac{\partial a_{k}(\mathbf{X})}{\partial x_{i}}=\left(\frac{\partial^{2} a_{k}}{\partial x_{i} \partial x_{j}}\right)_{i, j \in[1, k]}(\mathbf{X}) ; \\
& \frac{1}{(\ln 2)^{2}}\left(\frac{\partial^{2} a_{k}}{\partial x_{i} \partial x_{j}}\right)(\mathbf{X})=2^{x_{1}+\ldots+x_{r}} a_{k-r}\left(x_{r+1}, \ldots, x_{k}\right), \\
& r=\max (i, j) .
\end{aligned}
$$


Properties (A), (B) of the submanifolds $\mathbf{T}^{0}, \mathbf{T}^{+}, \mathbf{T}^{-}$follow immediately from the character of the first derivative $a_{k}(\mathbf{X})$. To prove $(\mathrm{C})$, consider the second differential of the function $a_{k}$, the quadratic form

$$
\begin{aligned}
& d^{2} a_{k}(\mathbf{X})=\sum_{i, j \in[1, k]} \frac{\partial^{2} a_{k}}{\partial x_{i} \partial x_{j}}(\mathbf{X}) d x_{i} d x_{j} \\
& =(\ln 2)^{2} \sum_{r \in[1, k]} 2^{x_{1}+\ldots+x_{r}} a_{k-r}\left(x_{r+1}, \ldots, x_{k}\right)\left(\sum_{i, j \in[1, r]} d x_{i} d x_{j}\right) \\
& =(\ln 2)^{2} \sum_{r \in[1, k]} 2^{x_{1}+\ldots+x_{r}} a_{k-r}\left(x_{r+1}, \ldots, x_{k}\right)\left(d x_{1}+\ldots+d x_{r}\right)^{2}>0 .
\end{aligned}
$$

\section{References}

A. Baker and G. Wüstholz [1993], Logarithmic forms and group varieties, J. Reine Angew. Math. 442, 19-62.

E. G. Belaga and M. Mignotte [1998], Embedding the $3 x+1$ conjecture in a $3 x+d$ context, Experiment. Math. 7, 145-151.

E. G. Belaga and M. Mignotte [2000], Cyclic structure of dynamical systems associated with $3 x+d$ extensions of Collatz problem, preprint 2000/018, Univ. Louis Pasteur, Strasbourg; http://www-irma.u-strasbg.fr/irma/publications/2000/00018.ps.gz.

J. C. Lagarias [1985], The $3 x+1$ problem and its generalizations, Amer. Math. Monthly $92,3-23$.

J. C. Lagarias [1990], The set of rational cycles for the $3 x+1$ problem, Acta Arith. 56, 33-53.

M. Laurent, M. Mignotte et Yu. Nesterenko [1995], Formes linéaires en deux logarithmes et déterminants d'interpolation, J. Number Theory 55, 285-321.

G. J. Wirsching [1998], The Dynamical System Generated by the $3 n+1$ Function, Lecture Notes in Math. 1681, Springer, Berlin.

Université Louis Pasteur

7, rue René Descartes

F-67084 Strasbourg Cedex, France

E-mail: belaga@math.u-strasbg.fr

Received on 9.11.2001

and in revised form on 28.2.2002 